\title{
Video Article \\ Detection of Invasive Pulmonary Aspergillosis in Haematological Malignancy Patients by using Lateral-flow Technology
}

\author{
Christopher Thornton ${ }^{1}$, Gemma Johnson ${ }^{2}$, Samir Agrawal $^{3}$ \\ ${ }^{1}$ Biosciences, University of Exeter \\ ${ }^{2}$ BICMS, Queen Mary University of London \\ ${ }^{3}$ St. Bartholomew's Hospital and The London NHS Trust \\ Correspondence to: Christopher Thornton at C.R.Thornton@ex.ac.uk
}

URL: https://www.jove.com/video/3721

DOI: doi:10.3791/3721

Keywords: Immunology, Issue 61, Invasive pulmonary aspergillosis, acute myeloid leukemia, bone marrow transplant, diagnosis, monoclonal antibody, lateral-flow technology

Date Published: 3/22/2012

Citation: Thornton, C., Johnson, G., Agrawal, S. Detection of Invasive Pulmonary Aspergillosis in Haematological Malignancy Patients by using Lateral-flow Technology. J. Vis. Exp. (61), e3721, doi:10.3791/3721 (2012).

\section{Abstract}

Invasive pulmonary aspergillosis (IPA) is a leading cause of morbidity and mortality in haematological malignancy patients and hematopoietic stem cell transplant recipients ${ }^{1}$. Detection of IPA represents a formidable diagnostic challenge and, in the absence of a 'gold standard', relies on a combination of clinical data and microbiology and histopathology where feasible. Diagnosis of IPA must conform to the European Organization for Research and Treatment of Cancer and the National Institute of Allergy and Infectious Diseases Mycology Study Group (EORTC/MSG) consensus defining "proven", "probable", and "possible" invasive fungal diseases ${ }^{2}$. Currently, no nucleic acid-based tests have been externally validated for IPA detection and so polymerase chain reaction (PCR) is not included in current EORTC/MSG diagnostic criteria.

Identification of Aspergillus in histological sections is problematic because of similarities in hyphal morphologies with other invasive fungal pathogens ${ }^{3}$, and proven identification requires isolation of the etiologic agent in pure culture. Culture-based approaches rely on the availability of biopsy samples, but these are not always accessible in sick patients, and do not always yield viable propagules for culture when obtained.

An important feature in the pathogenesis of Aspergillus is angio-invasion, a trait that provides opportunities to track the fungus immunologically using tests that detect characteristic antigenic signatures molecules in serum and bronchoalveolar lavage (BAL) fluids. This has led to the development of the Platelia enzyme immunoassay (GM-EIA) that detects Aspergillus galactomannan and a 'pan-fungal' assay (Fungitell test), that detects the conserved fungal cell wall component $(1 \rightarrow 3)$ - $\beta$-D-glucan, but not in the mucorales that lack this component in their cell walls 1,4 Issues surrounding the accuracy of these tests ${ }^{1,4-6}$ has led to the recent development of next-generation monoclonal antibody (MAb)-based assays that detect surrogate markers of infection ${ }^{1,5}$.

Thornton ${ }^{5}$ recently described the generation of an Aspergillus-specific MAb (JF5) using hybridoma technology and its use to develop an immuno-chromatographic lateral-flow device (LFD) for the point-of-care (POC) diagnosis of IPA. A major advantage of the LFD is its ability to detect activity since MAb JF5 binds to an extracellular glycoprotein antigen that is secreted during active growth of the fungus only ${ }^{5}$. This is an important consideration when using fluids such as lung BAL for diagnosing IPA since Aspergillus spores are a common component of inhaled air. The utility of the device in diagnosing IPA has been demonstrated using an animal model of infection, where the LFD displayed improved sensitivity and specificity compared to the Platelia GM and Fungitell $(1 \rightarrow 3)-\beta$-D-glucan assays ${ }^{7}$.

Here, we present a simple LFD procedure to detect Aspergillus antigen in human serum and BAL fluids. Its speed and accuracy provides a novel adjunct point-of-care test for diagnosis of IPA in haematological malignancy patients.

\section{Video Link}

The video component of this article can be found at https://www.jove.com/video/3721/

Protocol

\section{Collection, Storage and Preparation of Serum and Bronchoalveolar Lavage Fluids}

1. Collect serum from untreated blood samples by allowing blood to clot at $4{ }^{\circ} \mathrm{C}$, and store serum as aliquots at $-20^{\circ} \mathrm{C}$ prior to use. Bronchoalveolar lavage fluids (BAL) should also be stored as aliquots at $-20^{\circ} \mathrm{C}$. Note: Storage of serum and BAL as aliquots limits the potential for degradation of the target antigen by repeated freeze-thawing of samples during repeat testing.

2. Thaw samples and mix the serum and BAL samples thoroughly by vortexing and centrifuge for $1 \mathrm{~min}$ at $14,000 \mathrm{rpm}$. 
3. For routine testing of human serum samples, dilute the serum $1: 1(\mathrm{v} / \mathrm{v})$ with tissue culture medium (TCM). TCM consists of RPMI-1640 medium, $10 \%(\mathrm{v} / \mathrm{v})$ fetal calf serum, $1 \%(\mathrm{v} / \mathrm{v})$ of $200 \mathrm{mM}$ L-glutamine solution, and sodium azide $(0.02 \% \mathrm{w} / \mathrm{v})$ as preservative. The medium is prepared in advance and can be stored at $4{ }^{\circ} \mathrm{C}$ for several months. Apply $100 \mu$ of the diluted serum to the LFD.

4. For human BAL fluids, and for BAL fluids from animal models, apply $100 \mu \mathrm{l}$ of neat sample to the LFD, with no pre-treatment.

5. For serum from animal models, dilute serum $1: 2(\mathrm{v} / \mathrm{v})$ with phosphate buffered saline containing $4 \%(\mathrm{w} / \mathrm{v})$ EDTA, heat for $3 \mathrm{~min}$ in a boiling water bath, centrifuge for $5 \mathrm{~min}$ at $14,000 \mathrm{rpm}$, and apply $100 \mu \mathrm{l}$ of neat supernatant to the LFD device.

\section{Application of Serum and BAL to the Lateral-flow Device}

1. Store the lateral-flow devices at room temperature $\left(23^{\circ} \mathrm{C}\right)$. At this temperature, devices are stable for 12 months. Remove the devices from their pouches and place on a level surface.

2. Using a sterile pipette tip, apply $100 \mu \mathrm{l}$ of pre-treated serum or neat BAL sample to the release port of the device.

3. Allow the assay to run for $15 \mathrm{~min}$ at room temperature, at which time the results of the tests should be recorded. Note: Within seconds the fluid will be seen to migrate by capillary action along the nitrocellulose membrane in the observation window.

\section{Recording and Interpreting LFD Results}

1. The LFD consists of an internal control line (indicated by the letter $\mathrm{C}$ on the plastic housing) and a test line (indicated by the letter $\mathrm{T}$ ). The control line should always appear irrespective of Aspergillus antigen in the serum or BAL sample. This shows that the assay has run correctly.

2. If the Aspergillus antigen is present in the serum or BAL sample, the test line will also appear within $15 \mathrm{~min}$ of sample application. Because the intensity of the test line is proportional to the amount of Aspergillus antigen present in the sample, the test line can appear as a weak positive $(+)$, a moderate positive $(++)$ or as a strong positive $(+++)$. However, any positive test line, regardless of intensity, would indicate the presence of Aspergillus antigen in the sample. In the absence of Aspergillus antigen, no test line will appear, and the result is recorded as negative (-).

\section{Representative Results}

Representative examples of negative, weak positive, moderate positive, and strong positive LFD results with BAL and serum samples are shown in Figure 1.

Results of antigen-positive LFD tests (weak and strong) or negative LFD tests using BAL fluids from acute myeloid leukemia (AML) patients diagnosed according to EORTC/MSG diagnostic criteria are shown in Table 1. Included in this table are the corresponding clinical and mycological (galactomannan and culture) data, and results of an Aspergillus-specific PCR test developed at St. Bartholomews Hospital ${ }^{8}$, for each patient. Diagnosis of disease was based on host factors (neutropenia, prolonged use of corticosteroids, treatment with other recognized T-cell immunosuppressants), clinical criteria and GM positivity for BAL (here defined as a GM index value > 0.8). Under the 2002 EORTC/MSG guidelines $^{10}$, patients 12,13 and 16 were diagnosed with 'possible' IA on the basis of host factors and clinical criteria or GM positivity. According to the revised (2008) EORTC/MSG guidelines ${ }^{2}$, host factors and GM positivity alone or host factors and clinical criteria alone would not indicate 'possible' invasive fungal infection unless accompanied by supporting evidence from clinical data and mycology respectively. Patient 6 was diagnosed with 'probable' IA under both 2002 and 2008 guidelines because of host factors, major and minor clinical features and GM positivity. Note, that while neither the LFD nor PCR assays are currently included in EORTC/MSG guidelines, there is strong agreement between the two assays and the commercial galactomannan test indicating the presence of Aspergillus antigen and nucleic acid in the BAL samples of patients 6 and 12. Additional results of trials demonstrating the efficacy of the LFD and PCR assays in diagnosing IPA can be found in Johnson et al ${ }^{8}$. 


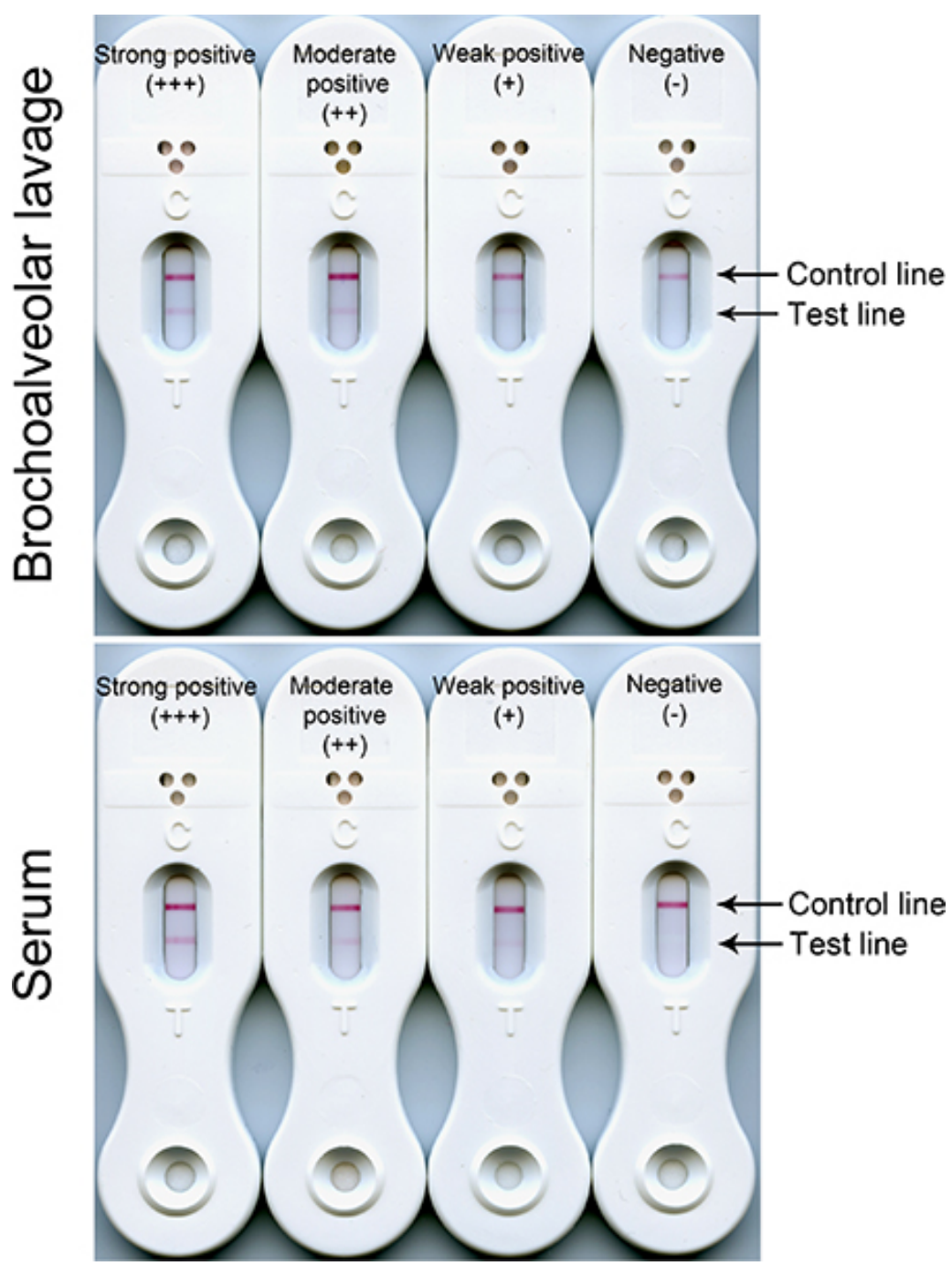

Figure 1. Negative (control line only) and positive (control and test line) results of LFD tests using serum and BAL. The intensities of the test line reactions are proportional to the concentrations of the target antigen in the serum and BAL samples. Reactions typically range from weak $(+)$ through moderate $(++)$ to strong $(+++)$. Regardless of test line intensity, all three serum positive reactions would indicate invasive pulmonary aspergillosis disease due to the presence of circulating Aspergillus antigen in the bloodstream. Positive BAL reactions (weak, moderate or strong) would indicate germination of spores and development of potentially pathogenic hyphae in the lungs. 


\begin{tabular}{|c|c|c|c|c|c|c|c|c|}
\hline Patient no. & \begin{tabular}{|l|} 
Patient \\
information
\end{tabular} & $\begin{array}{l}\text { Clinical } \\
\text { criteria }^{1}\end{array}$ & BAL culture $^{2}$ & $\begin{array}{l}\text { GM EIA } \\
\text { index } \\
\end{array}$ & $\begin{array}{l}\text { EORTC / MSG } \\
(2002)^{4}\end{array}$ & \begin{tabular}{|l|} 
EORTC / MSG \\
$(2008)^{5}$
\end{tabular} & \begin{tabular}{|l|} 
Aspergillus \\
PCR result
\end{tabular} & LFD result \\
\hline 6 & - & $\begin{array}{l}\text { Major CT } \\
\text { signs (nodule } \\
\text { and halo) and } \\
\text { one minor }\end{array}$ & Negative & 0.9 (positive) & Probable & Probable & Positive & $\begin{array}{l}\text { Weak positive } \\
(+)\end{array}$ \\
\hline 12 & $\begin{array}{l}\text { Presumed } \\
\text { previous } \\
\text { fungal } \\
\text { infection }\end{array}$ & $\begin{array}{l}\text { No major, one } \\
\text { minor }\end{array}$ & $\begin{array}{l}\text { Candida } \\
\text { glabrata }\end{array}$ & $\begin{array}{l}6.43 \text { (strong } \\
\text { positive) }\end{array}$ & Possible & None & Positive & $\begin{array}{l}\text { Strong positive } \\
(+++)\end{array}$ \\
\hline 13 & \begin{tabular}{|l|} 
Coagulase- \\
negative \\
Staphylococcus \\
and E. coli in \\
blood
\end{tabular} & $\begin{array}{l}\text { No major, two } \\
\text { minor }\end{array}$ & Negative & $\begin{array}{l}0.25 \\
\text { (negative) }\end{array}$ & Possible & None & Negative & Negative (-) \\
\hline 16 & Chest infection & $\begin{array}{l}3 \text { minor criteria } \\
\text { including new } \\
\text { infiltrate plus } \\
\text { plural effusion }\end{array}$ & Negative & $\begin{array}{l}0.16 \\
\text { (negative) }\end{array}$ & Possible & None & Negative & Negative (-) \\
\hline
\end{tabular}

${ }^{1}$ Nodules or halos on a computed tomography scan is suggestive of fungal infection

${ }^{2}$ Candida glabrata in BAL fluid would be regarded as a contaminant as it is the second most common yeast isolated as part of normal human flora $^{9}$

${ }^{3} \mathrm{An}$ index of $>0.8$ in the GM EIA test of BAL is indicative of Aspergillus infection

${ }^{4}$ Based on the 2002 EORTC/MSG diagnostic criteria for 'possible', 'probable' or 'proven' invasive fungal disease ${ }^{10}$

${ }^{5}$ Based on the revised (2008) EORTC/MSG diagnostic criteria for 'possible', 'probable' or 'proven' invasive fungal disease ${ }^{2}$

Table 1. Results of LFD tests of BAL samples from acute myeloid leukemia patients with probable IPA and from control AML patients (no evidence of infection), and EORTC/MSG diagnosis of infection.

\section{Discussion}

Definitive identification of IPA can only truly be achieved by isolation of the etiologic agent from biopsy samples, but recovery of suitable samples is often not possible in very sick patients and Aspergillus is rarely recoverable from blood. While major advances have been made in the use of computed tomographic scanning of the chest in IPA diagnosis, characteristics that are suggestive of pulmonary IPA such as the "halo" or "air-crescent" signs are either transient or can be attributed to breathing artifacts or other fungal infections ${ }^{11,12}$. Such data are therefore supplemented with serological techniques that aim to identify signature molecules (GM and $\beta$-glucan) from fungi that are circulating in the patient's serum or that are present in BAL fluids, sputum or urine samples ${ }^{13}$. While these tests display satisfactory sensitivity, they lack sufficient specificity or suffer from interference under certain conditions $s^{1,6}$.

The LFD test for IPA detection presented here enables the 'point-of-care' diagnosis of IPA and exploits technology that has been used to date in tests for the detection of viruses, bacteria, parasites and toxins ${ }^{14-19}$ and, most famously, for the home pregnancy tests first introduced by Unipath in 1988. In the Aspergillus LFD described here, the Aspergillus-specific MAb JF5 is immobilized to a capture zone (the test line) on a porous nitrocellulose membrane. Anti-mouse immunoglobulin immobilized to the membrane in a separate zone served as an internal control (control line). On addition of serum or BAL fluid to the release port, MAb JF5-colloidal gold conjugate in the release pad binds to the target antigen and the complex then passes along the porous membrane by capillary action. MAb JF5 immobilized in the capture zone binds to the JF5-colloidal gold-antigen complex resulting in a red test line. Any unbound JF5-colloidal gold conjugate binds to the internal control indicating that the assay has run correctly. This results in a red control line.

The test is quick, taking only 15 minutes to perform, is cheap compared to serum and BAL tests based on GM and $\beta$-glucan detection, and does not require expensive equipment or extensive laboratory facilities to run. Furthermore, MAb JF5 does not cross-react with the drugs or contaminants that have been shown to cause false-positive reaction in the $\mathrm{GM}$ and $\beta$-glucan tests ${ }^{1,4,6}$. An additional major advantage over current diagnostic tests is the LFDs ability to detect activity that is indicative of invasive growth of Aspergillus species.

A critical step in the LFD procedure is the need to read the results 15 min after application of the serum or BAL sample to the device. The test should not be left for longer than 15 min before recording the results, as this may bias result interpretation. A weak reaction will not be enhanced by extending the incubation period. Heat treatment of serum from animal models of infection has been found to improve assay sensitivity. A limitation of the test is that it is qualitative, and relies on the operator to make a subjective assessment of positivity. The intensity of the test line varies according to the antigen contents of serum and BAL samples (Figure 1). However, any positive reaction (determined by comparison to known negatives) indicates the presence of Aspergillus antigen and therefore infection. To limit the subjectivity of LFD assays, hand-held devices are available which allow quantification of LFD test line intensities and enable the establishment of threshold values for antigen detection ${ }^{20}$. 
Future developments of the LFD include its commercialization and the development of a multiplex LFD that allows simultaneous detection of other invasive fungal pathogens using highly specific MAbs ${ }^{3}$.

\section{Disclosures}

We have nothing to disclose.

\section{Acknowledgements}

Funding to Dr Thornton from Pfizer Limited is gratefully acknowledged.

\section{References}

1. Thornton, C.R. Detection of invasive aspergillosis. Adv. Appl. Microbiol. 70, 187-216 (2010).

2. De Pauw, B., Walsh, T. J., Donnelly, J. P., et al. Revised definitions of invasive fungal disease from the European Organization for Research and Treatment of Cancer/Invasive Fungal Infections Cooperative Group and the National Institute of Allergy and Infectious Diseases Mycology Study Group (EORTC/MSG) concensus group. Clin. Infect. Dis. 46, 1813-1821 (2008).

3. Thornton, C.R. Tracking the emerging human pathogen Pseudallescheria boydii by using highly specific monoclonal antibodies. Clin. Vacc. Immunol. 16, 756-764 (2009).

4. Pickering, J.W., et al. Evaluation of a $(1 \rightarrow 3)-\beta-D-g l u c a n$ assay for diagnosis of invasive fungal infections. J. Clin. Microbiol. 43, 5957-5962 (2005).

5. Thornton, C.R. Development of an immunochromatographic lateral-flow device for rapid serodiagnosis of invasive aspergillosis. Clin. Vacc. Immunol. 15, 1095-1105 (2008).

6. Verweij, P.E. \& Mennink-Kersten, M.A.S.H. Issues with galactomannan testing. Med. Mycol. 44, S179-183 (2006).

7. Wiederhold, N.P., et al. Comparison of lateral flow technology and galactomannan and $(1 \rightarrow 3)-\beta$-D-glucan assays for detection of invasive pulmonary aspergillosis. Clin. Vacc. Immunol. 16, 1844-1846 (2009).

8. Johnson, G.L., Bibby, D., Bustin, S., et al. Detection of Aspergillus in broncho-alveolar lavage fluid using two biological assays; evidence of active infection. Mycoses. 54 (Special Issue, Supplement 2), 130-131 (2011).

9. Malani, A., et al. Candida glabrata fungemia: experience in a tertiary care centre. Clin. Infect. Dis. 41, 975-981 (2005).

10. Ascioglu, S., et al. Defining opportunistic invasive fungal infection in immunocompromised patients with cancer and hematopoietic stem cell transplants: an international concensus. Clin. Infect. Dis. 34, 71-4 (2002).

11. Denning, D. Early diagnosis of invasive aspergillosis. Lancet. 355, 423-424 (2000).

12. Greene, R., Shibuya, K., \& Ando, T. In Aspergillus fumigatus and Aspergillosis , Latge, J.-P. \& Steinbach, W.J., Eds., $353-363$ (ASM Press, 2009).

13. Klont, R.R., Mennink-Kersten, M.A.S.H., \& Verweij, P.E. Utility of Aspergillus antigen detection in specimens other than serum samples. Clin Infect. Dis. 39, 1467-1474 (2004).

14. Iweala, O.I. HIV diagnostic tests: an overview. Contraception. 70, 141-147 (2004).

15. Ketema, F., Zeh, C., \& Edelman, D.C. Assessment of the performance of a rapid, lateral flow assay for the detection of antibodies to HIV. J. Acquir. Immune Defic. Syndr. 27, 63-70 (2001).

16. Moody, A. Rapid diagnostic tests for malaria parasites. Clin. Microbiol. Rev. 15, 66-78 (2002).

17. Parkash, O., et al. Performance of a lateral flow test for the detection of leprosy patients in India. J. Med. Microbiol. 57, 130-132 (2008).

18. Sharma, S.K., et al. Evaluation of lateral-flow Clostridium botulinum neurotoxin detection kits for food analysis. Appl. Environ. Microbiol. 71, 3935-3941 (2005).

19. Smits, H.L., et al. Lateral-flow assay for rapid serodiagnosis of human leptospirosis. Clin. Diagn. Lab. Immunol. 8, 166-169 (2001).

20. Faulstich, K., et al. in Lateral Flow Immunoassay, Wong, R., Tse, H., Eds, 157-185 (Humana Press, 2009). 